\title{
DNA damage accumulation and TRF2 degradation in atypical Werner syndrome fibroblasts with LMNA mutations
}

\author{
Bidisha Saha, Galynn Zitnik, Simon Johnson, Quyen Nguyen, Rosa A. Risques, George M. Martin \\ and Junko Oshima*
}

Department of Pathology, University of Washington, Seattle, WA, USA

\section{Edited by:}

Heidi A. Tissenbaum, University of

Massachusetts Medical School, USA

\section{Reviewed by:}

Stewart Frankel, University of

Hartford, USA

Giovanni Cenci, University of L'Aquila, Italy

${ }^{*}$ Correspondence:

Junko Oshima, Department of Pathology, University of Washington Box 357470, HSB K-543B, Seattle, WA 98195, USA

e-mail:picard@u.washington.edu
Segmental progeroid syndromes are groups of disorders with multiple features suggestive of accelerated aging. One subset of adult-onset progeroid syndromes, referred to as atypical Werner syndrome, is caused by mutations in the LMNA gene, which encodes a class of nuclear intermediate filaments, lamin A/C. We previously described rapid telomere attrition and accelerated replicative senescence in cultured fibroblasts overexpressing mutant lamin A. In this study, we investigated the cellular phenotypes associated with accelerated telomere shortening in LMNA mutant primary fibroblasts. In early passage primary fibroblasts with R133L or L140R LMNA mutations, shelterin protein components were already reduced while cells still retained telomere lengths comparable to those of controls. There was a significant inverse correlation between the degree of abnormal nuclear morphology and the level ofTRF2, a shelterin subunit, suggesting a potential causal relationship. Stabilization of the telomeres via the introduction of the catalytic subunit of human telomerase, hTERT (human telomerase reverse transcriptase), did not prevent degradation of shelterin components, indicating that reduced TRF2 in LMNA mutants is not mediated by short telomeres. Interestingly, $\gamma$ - $\mathrm{H} 2 \mathrm{AX}$ foci (reflecting double strand DNA damage) in early passage LMNA mutant primary fibroblasts and LMNA mutant hTERT fibroblasts were markedly increased in non-telomeric regions of DNA. Our results raise the possibility that mutant lamin $\mathrm{A} / \mathrm{C}$ causes global genomic instability with accumulation of non-telomeric DNA damage as an early event, followed by TRF2 degradation and telomere shortening.

Keywords: lamin A/C, telomeres, TRF2, progeroid syndromes, laminopathies

\section{INTRODUCTION}

Segmental progeroid syndromes are a genetically heterogeneous collection of disorders whose presentations resemble accelerated aging (Martin, 1978). The best known example of an adult-onset segmental progeroid syndrome is the Werner syndrome ("Progeria of Adult"; WS), caused by mutations in the WRN gene (Yu et al., 1996; Friedrich et al., 2010). Our International Registry of Werner syndrome (www.wernersyndrome.org/registry/registry) has accumulated several dozen cases of "atypical" WS (AWS) - cases submitted by clinicians to us as examples of WS, but lack WRN mutations and have normal levels of the WRN protein. A small subset of AWS cases are caused by LMNA mutations (Chen et al., 2003). The $L M N A$ gene encodes nuclear intermediate filaments, lamin A and lamin C, the major components of the nuclear lamina. These provide the nucleus with mechanical support and contribute to functional interactions with chromatin (Broers et al., 2006; Andres and Gonzalez, 2009). R133L and L140R are heterozygous dominant LMNA mutations located at the surface of a heptad repeat, suggesting that these mutations might perturb the intermolecular interactions of lamin A/C (Chen et al., 2003). There have been additional reports of heterozygous dominant LMNA mutations in AWS cases, including our recent report of heterozygous substitutions at the junction of LMNA exon 11 and intron 11 , which results in the generation of a very small amount of progerin (Hisama et al., 2011). Progerin is an aberrant splice form of lamin A; high levels are responsible for HutchinsonGilford progeria syndrome ("Progeria of Childhood"; HGPS; De Sandre-Giovannoli et al., 2003; Eriksson et al., 2003). In addition to progeroid syndromes, LMNA mutations are also responsible for muscular dystrophies, cardiomyopathies, partial lipodystrophies, and peripheral neuropathies (Broers et al., 2006).

Cells carrying LMNA mutations exhibit characteristic morphological nuclear abnormalities and altered mechanical properties of the lamina (Dahl et al., 2006). In addition to its structural roles, the nuclear lamina interacts with chromatin to participate in chromatin remodeling and heterochromatin organization, providing maintenance of nuclear organization, (Broers et al., 2006; Andres and Gonzalez, 2009), and thus influencing replication timing and transcription (Gonzalez-Suarez and Gonzalo, 2010; Hansen et al., 2010). In HGPS fibroblasts, changes in the lamina structure result in reorganization of heterochromatin and epigenetic changes in histones (Shumaker et al., 2006).

Cellular characteristics of human fibroblasts expressing progerin include genome instability such as increased $\gamma$-H2AX foci, continuous p53 activation (Liu et al., 2005; Scaffidi and Misteli, 2006; Kudlow et al., 2008; Musich and Zou, 2011), and defective cell proliferation, which can be partially overcome by inactivation of p53, ATM (ataxia telangiectasia mutated), 
and ATR (ATM- and RAD3-related) or introduction of human telomerase reverse transcriptase (hTERT; Liu et al., 2006; Kudlow et al., 2008). Furthermore, several studies have shown a role for A-type lamins in telomere maintenance (Gonzalez-Suarez et al., 2009; Benson et al., 2010). In primary fibroblasts derived from HGPS patients, DNA damage foci were shown to be localized to the telomeres in association with accelerated replicative senescence (Benson et al., 2010). hTERT immortalization of HGPS fibroblasts has been shown to lead to a reduction of p53 pathway signaling, further supporting the idea that the telomere may be a major DNA target of progerin (Benson et al., 2010). However, in an independent study, hTERT failed to prevent the accumulation of DNA damage upon induction of exogenous progerin expression (Scaffidi and Misteli, 2008). Studies of LMNA knockout mice provided evidence that lamin A/C interacts with telomeres and maintains the distribution of telomeres throughout the nucleus (Gonzalez-Suarez et al., 2009). Altered distributions of telomeres in LMNA knockout mouse cells were associated with deprotection and hyper-recombination of telomeric DNA, resulting in accelerated telomere loss and a DNA damage response (Gonzalez-Suarez et al., 2009). This suggests that, depending on the nature of the LMNA mutation(s), altered lamin $\mathrm{A} / \mathrm{C}$ may lead to accelerated telomere shortening via different mechanisms.

We previously demonstrated that human fibroblasts which over-express either the wild type lamin A, the mutant lamin A found in HGPS patients (delta 35 and delta 50), or the lamin A mutations in AWS patients (R133L and L140R), all undergo accelerated telomere attrition and rapid replicative senescence (Huang etal., 2008). Unlike the classical deletions that cause HGPS, amino acid substitutions of lamin A/C, such as those found in patients with AWS, have not been well studied for their effects on telomeres. It has been well established that telomeres become dysfunctional through replicative telomere attrition, inhibition of shelterin or the activation of oncogenes (Denchi and de Lange, 2007; Sfeir and de Lange, 2012; Suram et al., 2012). In this study, we investigated the cellular phenotypes associated with accelerated telomere shortening in AWS fibroblasts focusing on the shelterin complex. The shelterin complex protects chromosomal ends from erosions and consists of six major proteins, TRF2, TRF1, POT1, TPP1, Rap1, and TIN2 (Palm and de Lange, 2008). The POT1-TPP1 complex competes with the CST (CTC1, STN1, and TEN1) complex which terminates telomerase activity (Chen et al., 2012). Among shelterin components, TRF2 in particular is thought to play an important role in the protection of telomeres from erosion by binding to telomeric repeats to form a t-loop (Palm and de Lange, 2008). Loss of TRF2 protein expression or activity was found to trigger telomere shortening, the DNA damage response, chromosomal instability, and replicative senescence (Oh et al., 2003; Denchi and de Lange, 2007; Okamoto et al., 2013). TRF2 was shown to co-precipitate with lamin A (Luderus et al., 1996) and also to interact with lamin-associated proteins, LAP- $2 \alpha$ and BAF, in a cell cycle dependent manner (Dechat et al., 2004). In the present study we observed significantly reduced levels of TRF2 and other shelterin proteins in LMNA mutant AWS cells. We suggest that this reduction in protein levels is due to the degradation of TRF2 protein. Using immuno-fluorescent in situ hybridization (immuno-FISH) analysis we found that LMNA mutant cells accumulate increased levels of non-telomeric DNA damage in LMNA mutant hTERT cells. We therefore suggest that these forms of mutant lamin A induce genomic DNA damage and that such damage may contribute to the reduction of TRF2 proteins via activation of the p53-mediated signaling cascades, leading to the degradation of shelterin proteins. Suboptimum shelterin-telomere interactions may further lead to a loss of protection of telomeric ends and subsequent telomere attrition.

\section{MATERIALS AND METHODS ETHICS STATEMENT}

Patients signed approved consent forms to participate in the studies of the International Registry of Werner Syndrome (Seattle, WA, USA) prior to the initiation of the study. This study was approved by the Human Subjects Division, Institutional Review Board of the University of Washington, Seattle, WA, USA.

\section{CELL LINES AND CELL CULTURE}

Human diploid fibroblast cell lines were established from biopsies of skin samples. Cell lines 82-6 and 88-1 (normal) were obtained from newborn foreskin and the abdominal skin of a 62 year old female, respectively. Fibroblasts were cultured in Dulbecco's modified Eagle's medium (DMEM; Invitrogen, Carlsbad, CA, USA) supplemented with 10\% heat-inactivated fetal bovine serum (FBS; Thermo Scientific Hyclone, OH, USA) and penicillin and streptomycin (Invitrogen), as previously described (Chen et al., 2003). The media was changed every 3-4 days. All cells were cultured at $37^{\circ} \mathrm{C}$ in $5 \% \mathrm{CO}_{2}$. Primary fibroblasts were immortalized with a construct encoding hTERT (Rubio et al., 2002). MG-132 (10 $\mathrm{MM}$, Calbiochem, La Jolla, CA, USA) and cycloheximide (100 $\mu \mathrm{g} / \mathrm{ml}$, Sigma, St. Louis, MO, USA) was used when treating fibroblasts.

\section{BrdU INCORPORATION ASSAY}

To determine the fraction of proliferating cells, a 5-bromo- $2^{\prime}$ deoxyuridine (BrdU) incorporation assay was carried out using a BrdU labeling and detection kit (11 296736 001, Roche Applied Science, Indianapolis, IN, USA) according to the manufacturer's instructions. Briefly, primary fibroblasts from control, LMNA mutant and WRN mutant cell lines were grown for $24 \mathrm{~h}$ on cover slips. Cells were then labeled with $\operatorname{BrdU}(10 \mu \mathrm{M})$ for 24 or $48 \mathrm{~h}$, fixed in $70 \%$ ethanol with $50 \mathrm{mM}$ glycine for $20 \mathrm{~min}$ at $-20^{\circ} \mathrm{C}$ and processed for immunofluorescence. Cells were then incubated with the primary antibody, anti-BrdU (mouse monoclonal antibody, clone BMG 6H8 IgG1, 1:10 dilution) for $30 \mathrm{~min}$ at $37^{\circ} \mathrm{C}$ followed by Alexa Fluor 594-conjugated goat anti-mouse antibody (1:200 dilution; A-11005, Molecular Probes, Eugene, OR, USA) for $30 \mathrm{~min}$ at $37^{\circ} \mathrm{C}$. Cells were mounted onto slides using Vectashield with DAPI (Vectashield, Burlingame, CA, USA) and observed and photographed with Nikon Upright (Nikon Eclipse E600) microscope at the Keck Center for Imaging, University of Washington, Seattle, WA, USA. The percentages of cells with BrdUlabeled nuclei were determined by counting 300 cells per data point. 


\section{WESTERN BLOT ANALYSIS}

For western blot analyses, cells were lysed in $2 \times$ Laemmli buffer $[0.5 \mathrm{M}$ Tris $\mathrm{pH} 6.8,10 \%$ sodium dodecyl sulfate (SDS) glycerol]. Fifteen micrograms of total protein isolated from primary fibroblasts or immortalized fibroblast cell lines were separated on NuPAGE 4-12\% gradient Bis-Tris gel (Invitrogen) and transferred to polyvinylidene fluoride (PVDF) membranes. The membranes were incubated with anti-TRF2 (IMG-124A, clone 4a794.15, Imgenex, San Diego, CA, USA and sc-32106, Santa Cruz Biotechnology Inc., Santa Cruz, CA, USA), anti-TRF1 (sc5596, Santa Cruz Biotechnology Inc), anti-POT1 (sc-27952, Santa Cruz Biotechnology Inc.), anti-TIN2 (IMG-282, clone 59B388.1, Imgenex), anti-hRap1 (IMG-289, Imgenex), anti-TPP1 (ab54685, Abcam Inc., Cambridge, MA, USA) at a dilution of 1:2000 or anti$\beta$-actin (clone AC-15, Sigma, St Louis, MO, USA) at a dilution of 1:40,000 in a milk blocking solution, then with corresponding biotinylated secondary antibodies against mouse, rabbit or goat IgG (Vector Laboratories, Burlingame, CA, USA). The reactions were visualized with western lightening chemiluminescence reagent (NEL100, Perkin Elmer, Waltham, MA, USA) according to the manufacturer's instruction. The bands corresponding to TRF2, TRF1, POT1, TIN2, hRap1, and TPP1 were quantified using TotalLab software (Newcastle, UK) and normalized against $\beta$-actin levels.

For the measurement of the TRF2 protein half-life, immortalized fibroblast cell lines from the control, LMNA mutant and WRN mutant were treated with cycloheximide $(100 \mu \mathrm{g} / \mathrm{ml})$ for 8-12 h. Total protein extracts were subjected to the western analysis of TRF 2 and $\beta$-actin as described above. After normalizing to $\beta$-actin levels, TRF2 levels were calculated in order to estimate the protein half-lives.

\section{QUANTITATIVE REAL TIME PCR}

Quantitative reverse transcription polymerase chain reaction (RTPCR) for TRF2 mRNA was performed using Taqman gene expression assay system (Hs00194619_m1, Applied Biosystem, Foster City, CA, USA) and normalized against mRNA level of GAPDH (Hs02758991_g1, Applied Biosystem). Total RNA was isolated from the cultured cell pellet using Qiagen RNA isolation kit (Qiagen, Valencia, CA, USA) following the manufacturer's instructions. Reverse transcription was carried out with $2 \mu \mathrm{g}$ isolated total RNA. Quantitative PCR was performed with a Rotor-Gene 3000 (Corbett Research, Sydney, Australia). The data analysis was performed as previously described (Huang et al., 2008).

\section{TELOMERE QUANTITATIVE POLYMERASE CHAIN REACTION}

Mean telomere lengths were determined by qPCR as previously described (Huang et al., 2008). Genomic DNA was isolated via a salting out method and qPCR was performed with a RotorGene 3000 (Corbett Research, Sydney, Australia). The data were analyzed as previously described (Huang et al., 2008). The intraassay and inter-assay variability (CV) for qPCR was 3 and 5\%, respectively.

\section{TELOMERASE REPEAT AMPLIFICATION PROTOCOL ASSAY}

Telomerase activity in hTERT-immortalized cell lines was measured using the TRAPEZE XL Telomerase detection kit
(S7707, Millepore, Billerica, MA, USA). Cells were lysed in ice-cold 3-[(3-cholamidopropyl)dimethylammonio]-1-propanesulfonate (CHAPS) lysis buffer for $30 \mathrm{~min} .2 \mu \mathrm{l}$ of the cell extract was analyzed for the telomerase repeat amplification protocol (TRAP) assay. In the first step telomeric repeats were added to a substrate oligonucleotide by telomerase; then, the extended products were amplified by Taq polymerase using PCR with TS and RP (reverse) primers, generating a ladder of products in 6 base increments. The PCR products were separated on a $10 \%$ nondenaturing polyacrylamide gel (PAGE). The gel was stained with SYBR Safe (S33102, Invitrogen) to visualize the TRAP ladder using FluorChem Q imaging system (Alpha Innotech, San Leandro, CA, USA).

\section{IMMUNO-FISH}

Immuno-fluorescent in situ hybridization was performed as described previously (O'Sullivan et al., 2004) with modifications. Cells were grown on coverslips overnight and then fixed the next day with 3.7\% paraformaldehyde in phosphate buffered saline (PBS; pH 7.4) plus $0.2 \%$ Triton X100 for $10 \mathrm{~min}$ at room temperature. Cells were blocked for $30 \mathrm{~min}$ at $37^{\circ} \mathrm{C}$ and incubated with primary antibody, mouse anti-TRF2 (IMG-124A, clone 4a794.15, Imgenex) or rabbit anti- $\gamma$-H2AX (2577, Cell Signaling Technologies, Beverly, MA, USA) for $30 \mathrm{~min}$ at $37^{\circ} \mathrm{C}$. They were then incubated with secondary antibody, donkey anti-mouse Alexa Fluor or donkey anti-rabbit Alexa Fluor (Invitrogen Molecular Probes, Eugene, OR, USA) for $1 \mathrm{~h}$ at room temperature, and then post-fixed with 3.7\% paraformaldehyde for $5 \mathrm{~min}$. After washing in PBS, cells were processed for FISH. Cells were treated with $100 \mu \mathrm{g} / \mathrm{ml}$ of RNase A (Sigma-Aldrich, St. Louise, MO, USA) in PBS for $45 \mathrm{~min}$. at $37^{\circ} \mathrm{C}$, then dehydrated in an ethanol series and air dried. Peptide nucleic acid (PNA) probe (200 nM, CentFAM, TelC-FITC, or TelC-Cy3; Panagene Inc., Daejeon, Korea) in hybridization buffer was placed on top of the coverslips, heated to $75-78^{\circ} \mathrm{C}$ for $15 \mathrm{~min}$, then removed, wrapped in foil and allowed to sit at room temperature overnight. The next day the coverslips were washed with $70 \%$ formamide in Tris buffer, washed with preheated $\left(55-60^{\circ} \mathrm{C}\right)$ Tween 20 solution, dehydrated in an ethanol series and then air dried. Coverslips were mounted on slides with DAPI (Vectashield) and sealed with clear nail polish.

\section{IMAGE PROCESSING AND ANALYSIS OF TELOMERE LENGTH, TRF2 INTENSITIES AND CO-LOCALIZATION}

The images were obtained in Z-series with Deltavision deconvolution microscope (Applied Precision, Issaquah, WA, USA) at the Keck Center (University of Washington, Seattle, WA, USA). The images were deconvolved using SoftWorx software (Applied Precision, Issaquah, WA, USA). They were analyzed for average telomere intensity (which is proportional to telomere length), and average TRF2 intensity using a software program, 4xColocalization, version 138, provided by the University of Washington Nathan Shock Center, as described previously (O'Sullivan et al., 2004). The images were also analyzed for co-localization (telomere and TRF2; telomere and $\gamma$-H2AX; TRF2 and $\gamma$-H2AX). Statistical significance was determined by the Student $t$-test (Chen et al., 2003; Huang et al., 2008). 


\section{NUCLEAR MORPHOLOGY AND CORRELATION ANALYSIS}

To assess the degree of nuclear irregularity, nuclear contour ratios (NCRs) were determined in randomly selected nuclei of LMNA mutant fibroblasts using MetaMorph software as described previously (Saha et al., 2010). The nuclei were also analyzed for average TRF2 intensities. The correlation between NCR and TRF2 intensities was analyzed using Pearson's correlation coefficient, $r$. For all experiments, a two-tailed $P$ value of $<0.05$ was considered significant.

\section{RESULTS REDUCTION OF SHELTERIN COMPONENTS IN PRIMARY LMNA MUTANT FIBROBLASTS}

We first analyzed the steady state protein expression levels of the shelterin subunits TRF2, TRF1, POT1, TIN2, TPP1, and Rap1 in primary fibroblasts carrying a heterozygous R133L mutation derived from Registry\# PORTU8010 and in primary fibroblasts carrying a heterozygous L140R mutation derived from Registry\# NORWAY1010 (Chen et al., 2003). The controls were two normal primary fibroblast cultures, 82-6 and 88-1 (Oshima et al., 1995; Huang et al., 2008) as well as cultures of WRN mutant fibroblasts, Registry\# MCI7885, derived from a patient with classical Werner syndrome (Oshima et al., 1995; Yu et al., 1996). The latter was included as a "control" since these fibroblasts undergo accelerated telomere shortening by different mechanisms, namely defects in lagging strand synthesis of telomeres (Crabbe et al., 2004). We examined fibroblasts in early passages (BrdU labeling index higher than $60 \%$ ) in order to detect proximal changes rather than changes secondary to telomere shortening and replicative senescence.

Western analysis showed that most subunits of the shelterin complex were reduced in both types of LMNA mutant fibroblasts (Figure 1A). Steady state levels of TRF2 were 46 and 45\%, POT1 was 57 and 56\%, TIN2 was 36 and 35\%, and Rap1 was 46 and $40 \%$, in $\mathrm{R} 133 \mathrm{~L}$ and $\mathrm{L} 140 \mathrm{R}$ mutants, respectively, compared to the control, 82-6 fibroblasts (Figure 1B). The other two components, TRF1 and TPP1, were also slightly reduced to 73 and $62 \%$ in R133L mutants and 74 and 92\% in L140R mutants (Table 1). Interestingly, WRN mutant fibroblasts also showed reduced protein expression of all of the shelterin components.

Since the length of the telomere determines the number of available binding sites for the shelterin complex, cells with short telomeres (i.e., late passage or senescent cells) would require and presumably express lower amounts of the shelterin components. To rule out this possibility, relative telomere lengths were measured by quantitation of fluorescence in situ hybridization images (QFISH), as established for normal fibroblasts undergoing replicative senescence (O'Sullivan et al., 2004). Using this method, we found that the telomere lengths of young LMNA mutant fibroblast cultures were comparable to those of controls after normalizing to centromere signal intensities (Figure 1C). Mean telomere lengths were also measured by quantitative PCR, showing no significant differences among LMNA mutant fibroblasts and controls (Figure 1C). The mean telomere lengths of the two controls were similar despite the difference in the age of donors, 82-6 (newborn) and 88-1 (adult; Figure 1C). This may be due to the fact that there is a wide range of mean telomere lengths and replicative life spans among normal individuals. The labeling index determined by the $48 \mathrm{~h}$ BrdU incorporation and population doubling (PD) of the culture were 94\% in 82-6 (PD 9), 89\% in 88-1 (PD 7), 71\% in R133L (PD 5), 96\% in L140R (PD 6), and 91\% in MCI7885 (PD 6), confirming that the LMNA mutant fibroblast cultures used in these experiments were indeed from young passages. This indicates that the reduction of shelterin proteins observed in LMNA mutants was not a direct result of shortened telomeres.

Next, we focused on the role of TRF2, a major component of the shelterin complex, in preventing telomere erosion. To determine whether the reduction of TRF2 proteins in LMNA mutant fibroblasts occurs via a transcriptional mechanism or posttranscriptional degradation, we analyzed TRF2 expression at the mRNA level. The qRT-PCR results showed that the steady state levels of TRF2 mRNA in LMNA mutant fibroblasts were not significantly different when compared to the controls (Figure 1D). These results suggest that the TRF2 protein may undergo degradation in LMNA mutant cells, resulting in low TRF2 protein levels while the cells still retain long telomeres.

\section{NUCLEAR MORPHOLOGICAL ABNORMALITIES CORRELATE WITH REDUCTIONS OF TRF2 WITHIN LMNA MUTANT CULTURES}

We previously reported that primary LMNA mutant fibroblasts, but not control fibroblasts or immortalized LMNA mutant cells, exhibit abnormal nuclear morphology, as assessed by NCR (Chen et al., 2003). In control fibroblasts, 82-6, the contour ratio was $0.842 \pm 0.036$, whereas in $\mathrm{R} 133 \mathrm{~L}$ the contour ratio was $0.749 \pm 0.118(P<0.005)$; for $\mathrm{L} 140 \mathrm{R}$, the ratio was $0.815 \pm 0.074$ $(P<0.05)$. We noted that within the LMNA mutant cultures, the cells with more distorted nuclei tended to have lower levels of TRF2 (Figure 2A). Quantitative analysis revealed a significant positive correlation between NCR and TRF2 protein level per nucleus in both R133L and L140R mutant cultures: Pearson's correlation coefficient, $r$, was 0.365 for R133L $(P<0.005)$ and was 0.379 for L140R $(P<0.05$; Table 2$)$. The presence of a correlation between and NCR and TRF2 levels supports the idea that abnormal lamin $\mathrm{A} / \mathrm{C}$ function, as reflected by abnormal nuclear morphology, may be causally related to the TRF2 degradation. As expected, control fibroblast cultures did not show significantly abnormal NCRs.

\section{INTRANUCLEAR LOCALIZATION OF TRF2 PROTEIN IN LMNA MUTANT CELLS}

Next, we assessed the intranuclear localization of TRF2 in relation to telomeres using immuno-FISH. Co-localization analysis of TRF2 and telomeres revealed that the fraction of signals of telomeric foci that overlapped with signals of TRF2 foci was slightly less in $L M N A$ mutant fibroblasts $66 \%$ in $\mathrm{R} 133 \mathrm{~L}$ and $-68 \%$ in L140R as compared to 78\% in the control, 82-6 (Figures 2B,C). Approximately 34\% $(P=0.06)$ of telomeric foci in the $\mathrm{R} 133 \mathrm{~L}$ mutant and $32 \%(P=0.174)$ in the L140R mutant were free of TRF2. These results agree with our western results, which showed a reduction of TRF2 protein in mass cultures. The TRF2 proteins in LMNA mutants, albeit reduced in amount, were co-localized to the telomeres (Figure 2B). These findings are consistent with the hypothesis that LMNA mutant fibroblasts in young passages might have lost optimum levels of shelterin complexes prior to telomere shortening, and that the reduced levels of TRF2 protein 


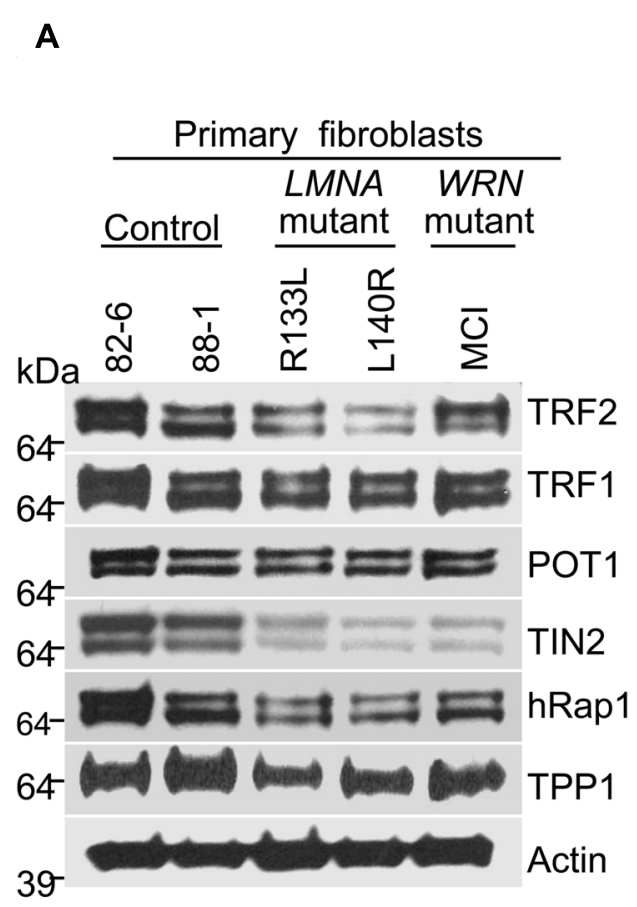

B

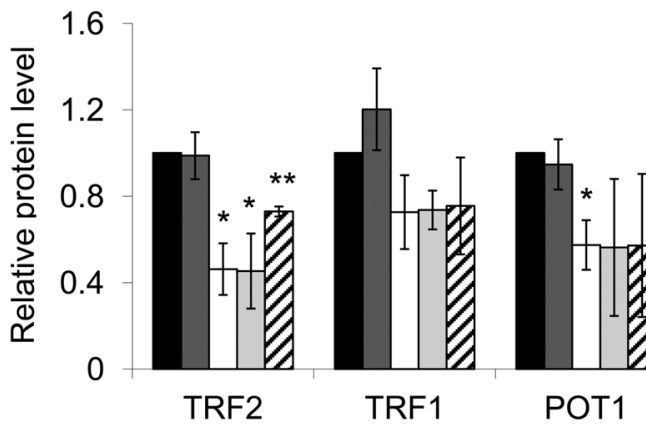

FIGURE 1 | Expression of shelterin subunits in LMNA mutant primary fibroblasts. (A) Western analysis of TRF2, TRF1, POT1, TIN2, hRap1, and TPP1 in control, LMNA and WRN mutant primary fibroblasts. Protein levels were normalized to $\beta$-Actin. Autoradiographies with exposures beyond the linear range are shown for clarification of bands. (B) Densitometric scanning of band intensities obtained from western analysis. Data are means \pm s.d. from three independent experiments. ${ }^{*} P<0.05$; ${ }^{*} P<0.01$; ${ }^{*} * P<0.001$. (C) Analysis of relative telomere lengths expressed as average telomere intensities \pm s.d. in indicated cell types of control, LMNA mutant and WRN
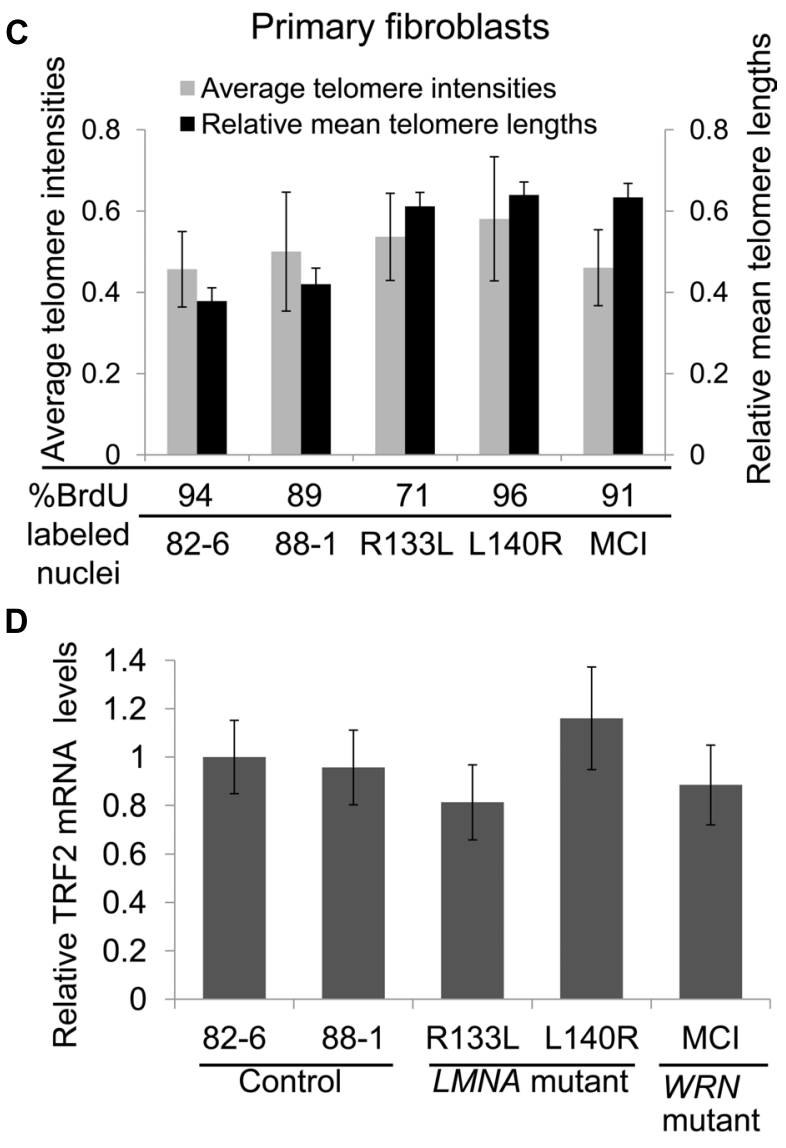

observed in LMNA mutants could be a causal step rather than a consequence of accelerated telomere attrition.

\section{R133L AND L140R MUTATIONS INDUCE ACCUMULATION OF GENOMIC DNA DAMAGE}

It has been reported that progerin induces a DNA damage response at the telomeres (Benson et al., 2010). We asked whether non-progerin producing LMNA mutations, R133L and L140R, also cause the accumulation of DNA damage and if so, where. To answer these questions, we assayed a marker of DNA damage, $\gamma$ $\mathrm{H} 2 \mathrm{AX}$ foci. In control fibroblasts, up to approximately $4 \%$ of cells were positive for $>5 \gamma$-H2AX foci (Figures 3A,B). The fraction of cells with $>5 \gamma$-H2AX foci was increased to $22 \%(P=0.06)$ in R133L and 36\% $(P=0.01)$ in L140R mutants (Figure 3B). 
Table 1 | Mean values of relative protein levels of shelterin components in primary and hTERT-immortalized fibroblasts of LMNA mutant cell lines. Protein levels in mutant lines were normalized to the average in controls, 82-6 and 82-6 hTERT.

\begin{tabular}{|c|c|c|c|c|}
\hline \multirow{3}{*}{$\begin{array}{l}\text { Shelterin } \\
\text { components }\end{array}$} & \multicolumn{4}{|c|}{ LMNA mutant cell lines } \\
\hline & \multicolumn{2}{|c|}{ PORTU8010 } & \multicolumn{2}{|c|}{ NORWAY 1010} \\
\hline & R133L & R133L hTERT & L140R & L140R hTERT \\
\hline TRF2 & 0.462 & 0.544 & 0.453 & 0.370 \\
\hline TRF1 & 0.726 & 0.429 & 0.736 & 0.362 \\
\hline POT1 & 0.574 & 0.491 & 0.562 & 0.470 \\
\hline TIN2 & 0.363 & 0.785 & 0.345 & 0.590 \\
\hline hRap1 & 0.460 & 0.590 & 0.398 & 0.312 \\
\hline TPP1 & 0.623 & 1.319 & 0.921 & 1.212 \\
\hline
\end{tabular}

To assess the degree to which the DNA damage foci in young passages of LMNA mutant cells were localized to the telomeres, we analyzed the co-localization of $\gamma-\mathrm{H} 2 \mathrm{AX}$ and telomeres using immuno-FISH (Figures 3A,C). In control cells, $\gamma$-H2AX signals were hardly visible as foci at standard exposures whereas in LMNA mutant cells $\gamma$-H2AX foci tended to be larger than those seen in the controls (Figure 3A). The majority of the $\gamma$ H2AX foci present in LMNA mutant cells were not co-localized to telomeric DNA (89\% in RI33L and 87\% in L140R; Figure 3C). Telomeric $\gamma$-H2AX represented relatively minor fractions of the total foci. These data indicate that, during early passages, R133L and L140R LMNA mutant fibroblasts may sustain non-telomeric genomic damage prior to the accumulation of damage at the telomeres.

\section{TELOMERE STABILIZATION DOES NOT PREVENT REDUCTION OF SHELTERIN EXPRESSION IN LMNA MUTANTS}

To further confirm that the reduction of shelterin complex proteins and the accumulation of DNA damage seen in primary LMNA mutant fibroblasts was not due to shortened telomeres, we analyzed TRF2 and other shelterin protein expressions in LMNA mutant and control fibroblasts immortalized with hTERT. Telomerase activity in all hTERT-immortalized cell lines was confirmed with a TRAP assay (data not shown). Western analysis showed an overall reduction of most of the shelterin subunit proteins in mutant hTERT lines in a pattern similar to what was observed in primary fibroblasts; TRF2, TRF1, POT1, TIN2, and Rap1 were reduced to $54,43,49,79$, and $59 \%$, respectively, in R133L mutant and 37, 36, 47, 59, and 31\%, respectively, in L140R mutant hTERT fibroblasts cell lines, as compared to control hTERT lines (Figure 4A; Table 1). Both Q-FISH analysis and quantitative PCR of the telomeres showed that the telomeres in LMNA mutant hTERT cells were not shorter than those of control hTERT lines (Figure 4B). These data indicate that the reduction of the shelterin complex subunits occurs without shortened telomeres in LMNA mutant hTERT lines, consistent with our findings in primary fibroblasts described above.
As observed in primary fibroblasts, TRF2 mRNA levels in the LMNA mutant hTERT lines were comparable to those of control hTERT lines, indicating that the reduction of TRF2 occurs post-transcriptionally (Figure 4C). In normal human diploid fibroblasts TRF2 undergoes rapid proteasome-mediated degradation after dissociation from the telomeres during replicative senescence (Zhou et al., 2009; Fujita et al., 2010). When proteasome degradation was inhibited by MG-132, TRF2 protein levels in R133L and L140R mutants were partially restored from 49 to $67 \%$ and 33 to $63 \%$, respectively (Figure 4D). This suggests that a part of the post-translational degradation of TRF2 in LMNA mutants is through proteasomal degradation. These data indicate that the stabilization of telomeres by hTERT does not prevent the degradation of TRF 2 and that there may be other mechanisms that initiate TRF2 reduction in LMNA mutant fibroblasts.

\section{LMNA MUTANT hTERT FIBROBLASTS ACCUMULATE NON-TELOMERIC GENOMIC DAMAGES}

To examine whether DNA damage is involved in the reduction of TRF2 in LMNA mutant cells with stable telomeres, we analyzed DNA damage foci in hTERT-immortalized LMNA mutant and control fibroblasts. The results showed that the fraction of cells positive for $>5 \gamma$-H2AX foci in LMNA mutant hTERT fibroblasts was decreased compared to the LMNA mutant primary fibroblasts. However, the number remained higher ( 9 and 14\% in R133L and L140R mutants, respectively) when compared to the control hTERT cell lines, which were devoid of cells with $>5 \gamma$-H2AX foci (Figure 5A). Co-localization analysis revealed that only 12 and 5\% of total $\gamma$-H2AX foci co-localized with TRF2 (a marker of telomeres) in R133L and L140R mutant cells, respectively, whereas the rest of the DNA damage signals were distributed to non-telomeric DNA (Figure 5B). These results indicate that mutant lamin A/C causes the accumulation of non-telomeric DNA damage and the reduction of TRF2 in hTERT-immortalized fibroblasts without substantial telomeric damage, as observed in young primary fibroblasts.

\section{DISCUSSION}

In this study we investigated the role of mutant lamin A in accelerating telomere shortening using LMNA mutant fibroblasts derived from AWS patients. We observed that the levels of TRF2, along with most of the other subunits of the shelterin complex, were significantly decreased in LMNA mutant fibroblast cells. TRF2 mRNA levels remained unchanged as compared to control cells with matched BrdU labeling indices. It should be emphasized that both mutant and control primary cells used in these experiments were in early passages, as evidenced by the labeling indices and the comparable lengths of the telomeres. This indicates that the reduction of TRF2 and other shelterin protein levels occurred prior to the shortening of the telomeres in LMNA mutant cells. The depletion in shelterin subunit protein levels in LMNA mutant primary fibroblasts was not corrected by introducing hTERT expression, but it was partially restored in response to proteasome inhibitor treatment. This supports our interpretation that the reduction of TRF2 in LMNA mutant fibroblasts is not merely secondary to the shortening of telomeres, but is primarily caused by LMNA mutations which lead to degradation of the TRF2 protein. In 


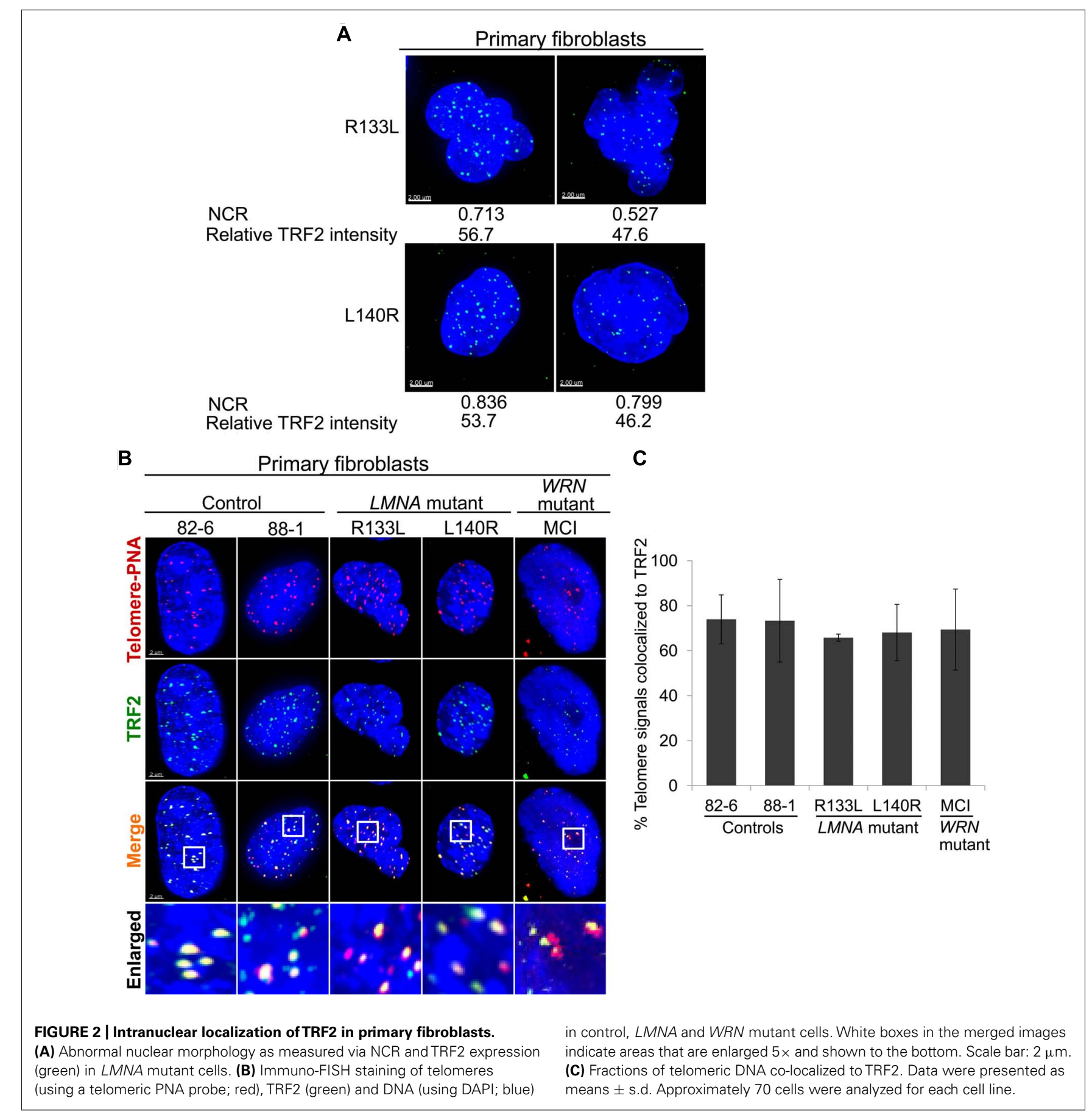

primary LMNA mutant cells, telomere damage and shortening contribute to further reduction of TRF2. In LMNA mutant cell cultures, the cells with more misshapen nuclei, as measured via NCR, exhibited lower TRF2 protein levels. This also suggests a causative role for mutant lamin A in the reduction of the TRF2 protein. In addition, LMNA mutant cells accumulated significant genomic DNA damage which persisted after ectopic expression of hTERT.

Based on the above findings, we propose that LMNA mutations first induce the accumulation of double stranded DNA damage.
This damage then leads to the activation of the p53 pathway, TRF2 degradation and telomere shortening (Figure 6). In this scenario, double stranded DNA damage accumulates in LMNA mutant cells in either telomeric or non-telomeric regions of the DNA, which in turn causes TRF2 degradation. The disruption of proper shelterin complex formation due to suboptimum levels of TRF2 and other shelterin components may then destabilize the telomeres, making them more vulnerable to nucleolytic degradation and accelerate telomere shortening and cellular senescence (Sfeir and de Lange, 2012). Our results showing the presence of 
Table 2 | Correlation analysis of nuclear morphological abnormality and reduction of TRF2 within LMNA mutant primary fibroblast cultures.

\begin{tabular}{lll}
\hline Cell Line & PORTU8010 & NORWAY1010 \\
\hline LMNA mutation & R133L & L140R \\
Nuclear contour ratio & $0.749 \pm 0.118$ & $0.815 \pm 0.074$ \\
Relative TRF2 intensity & $54.6 \pm 6.0$ & $51.5 \pm 5.3$ \\
Pearson's correlation coefficient $(r)$ & 0.365 & 0.379 \\
$P$ value & 0.003 & 0.01 \\
Number of cells examined & 65 & 40 \\
\hline
\end{tabular}

Nuclear contour ratios (NCR) were calculated as $4 \pi A / P^{2}$, where $P=$ Perimeter $A=$ Area. $N C R$ value $=1$, indicates a perfect circle. The correlation between NCR and TRF2 protein amount in cells was determined using Pearson's correlation analysis. Pearson's correlation coefficient $(r)$ is a number between -1 and $1 . r$ closer to 1 indicates a positive correlation, whereas $r$ closer to -1 indicates a negative correlation.

accumulated DNA damage in both LMNA mutant primary and hTERT fibroblasts are consistent with such a model (Figure 6, bold arrows).

Considering the size of telomeric DNA relative to that of the total genome, the number of double stranded DNA damage foci per unit of DNA sequence may be higher in telomeric DNA than in non-telomeric regions in primary LMNA mutant cells. However, there are substantially more DNA lesions in non-telomeric DNA, consistent with a major and, perhaps, primary impact on overall genomic instability. We speculate that the decrease in shelterin complex proteins might lead to the accumulation of damage at telomeres at later passages of primary cultures correlated with increased accumulation of mutant lamin A. TRF2 is also known to have an essential role in non-telomeric DNA damage repair, namely homologous recombination (Mao et al., 2007). It is therefore, possible, that depletion of TRF2 in LMNA mutant AWS cells may contribute to compromised double strand break repair and additional accumulation of DNA damage (Figure 6). The expression levels of the shelterin subunits were also reduced in WRN mutant fibroblasts. This can be explained, in part, by the accumulation of genomic DNA damage.

It is not known if the pattern or degree of DNA damage is different depending on the type of LMNA mutation. The presence of accumulated DNA damage in our LMNA mutant hTERT lines is in agreement with a previous study of progerin expressing cells by Scaffidi and Misteli (2006). In contrast, Benson et al. (2010), described the accumulation of DNA damage almost exclusively at the telomeres in HGPS cells, but not in hTERT HGPS cells. Additional research is required to explain these discrepant results.
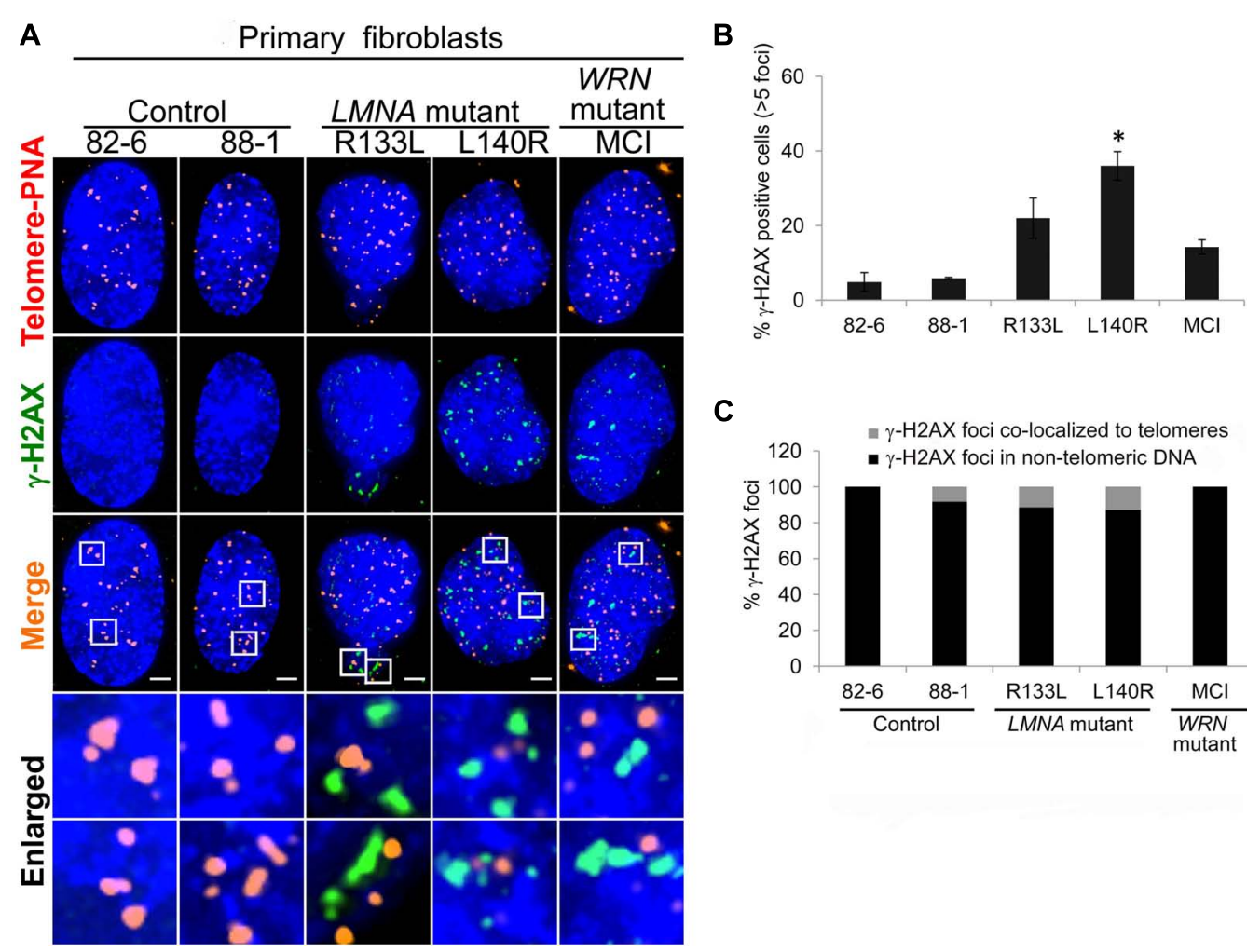

FIGURE 3 | Detection of $\boldsymbol{\gamma}$-H2AX DNA damage foci in LMNA mutant cells. (A) Immuno-FISH staining of telomeres (using a telomeric PNA probe; red), $\gamma$-H2AX (green), and DNA (using DAPI; blue) in control, LMNA mutant and WRN mutant cells. White boxes in the merged images indicate areas that are enlarged $5 x$ and shown to the bottom. Scale bar: $2 \mu \mathrm{m}$. (B) Percentages of cells with $>5 \gamma$-H2AX-labeled foci. Data are averages of three independent experiments. Bars represent \pm s.d. ${ }^{*} P<0.05$. (C) Quantitation of $\gamma-\mathrm{H} 2 \mathrm{AX}$ foci detected in (A). Signals of $\gamma-\mathrm{H} 2 \mathrm{AX}$ foci overlapping telomeres (gray) and those not overlapping (black) are shown separately. A minimum of 70 cells were analyzed for each cell line. Scale bar: $2 \mu \mathrm{m}$. 
A

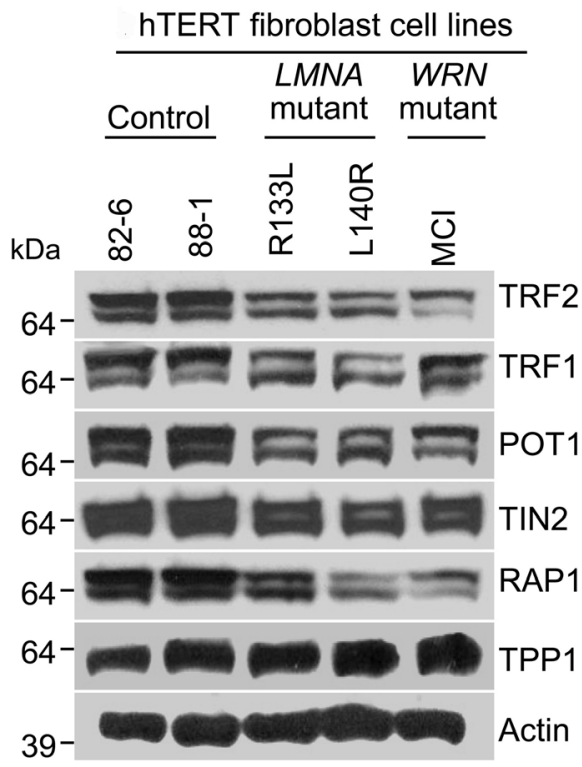

D
B
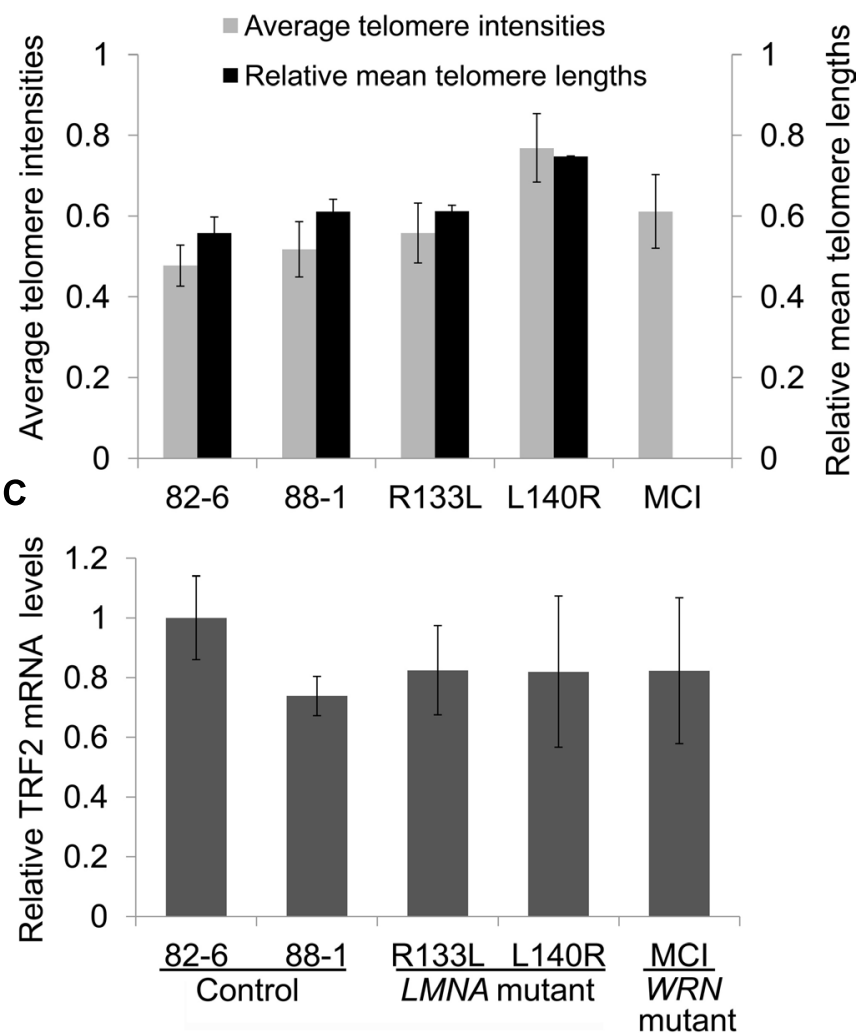

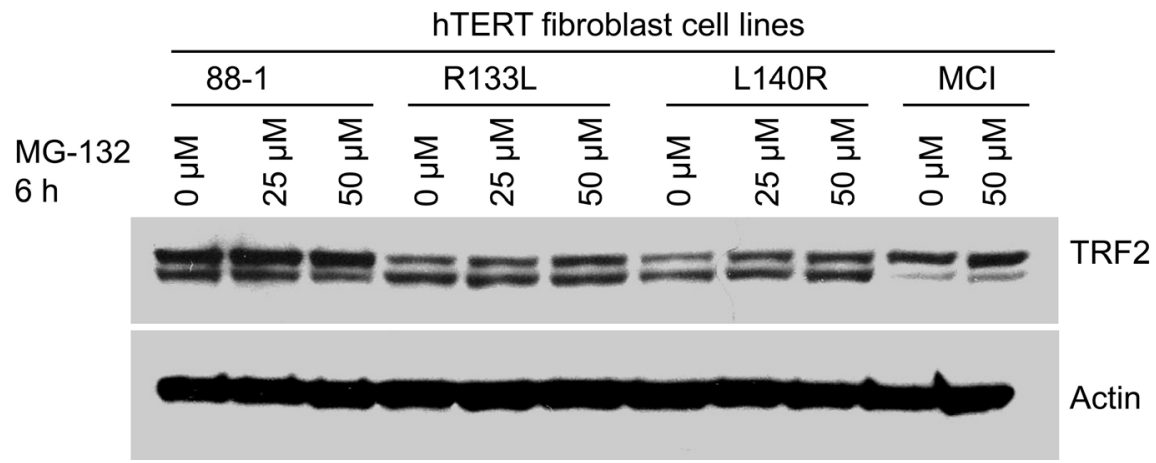

FIGURE 4 | Expression of shelterin subunits in hTERT-immortalized cell lines of control, LMNA mutant and WRN mutant fibroblasts. (A) Western analysis of TRF2, TRF1, POT1, TIN2, hRap1, and TPP1 in hTERT-immortalized fibroblasts of indicated cell lines. Autoradiographies with higher exposures are shown for clarification of bands. (B) Analysis of relative telomere lengths expressed as average telomere intensities \pm s.d. in indicated cell types, as shown in Figure 1. A minimum of 70 cells were analyzed for each cell lines. Relative mean telomere lengths ( \pm s.d.) were measured in hTERT-immortalized fibroblasts using qPCR. (C) TRF2 mRNA expression in
hTERT-immortalized normal, LMNA mutant and WRN mutant fibroblasts. TRF2 mRNA expression was detected by qRT-PCR. GAPDH mRNA was used as an internal standard. Data are presented as mean \pm s.d. from three independent experiments. The control (82-6) value was taken as onefold. (D) Expression of TRF2 protein after proteasome inhibition in LMNA mutant cells. hTERT-immortalized cell lines of control and LMNA mutant fibroblasts were incubated in the presence or absence of MG-132 for $6 \mathrm{~h}$ and TRF2 protein levels were detected using immunoblotting.
The p53-Siah1-TRF2 pathway was recently demonstrated to be a mechanism of TRF2 degradation in senescent normal fibroblasts with short telomeres (Fujita et al., 2010). Studies of doxorubicin treated colon cancer cells and lymphoblast cells provided evidence that non-telomeric DNA damage can also activate the p53-Siah1 pathway and result in proteosomal degradation of TRF2 (Fujita et al., 2010). Our observations of a preponderance of DNA damage in the non-telomeric genomic DNA of primary and LMNA mutant hTERT cells raise the possibility that this is the trigger leading to the activation of the 553 pathway and the degradation of TRF2 protein in LMNA mutant cells. We examined baseline phosphorylated p53 protein levels by western analysis (data not shown) which showed 
A

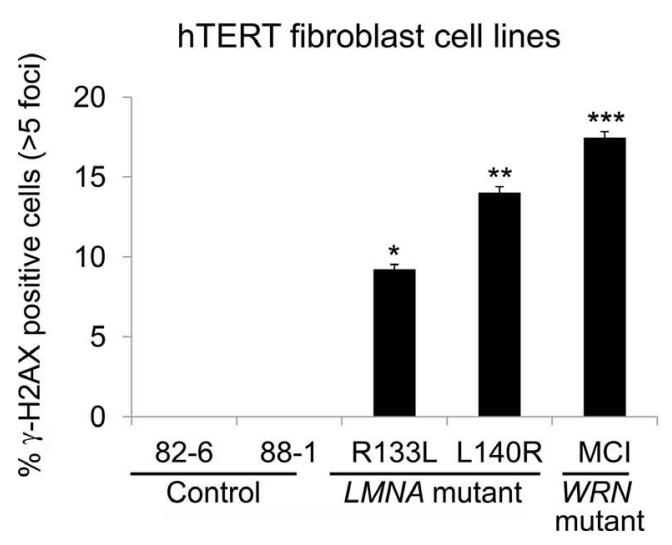

B

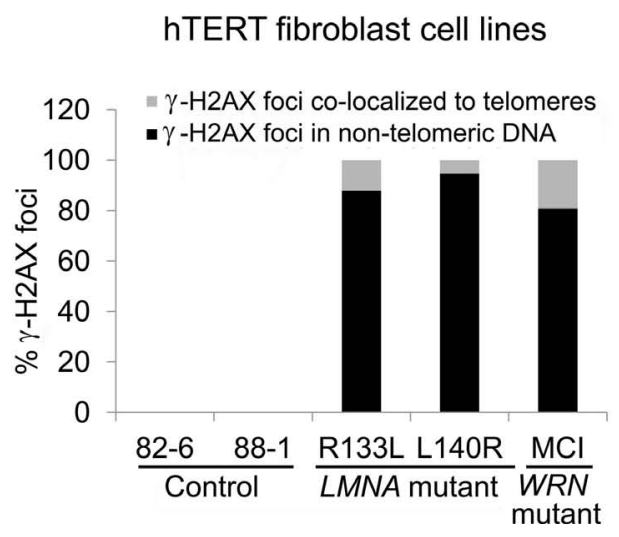

FIGURE 5 | Detection of $\gamma$-H2AX foci in control and LMNA mutant fibroblasts expressing hTERT. (A) Percentage of cells with $>5 \gamma-\mathrm{H} 2 \mathrm{AX}$ labeled foci, as stated in Figure 3. ${ }^{*} P<0.05,{ }^{* *} P<0.005,{ }^{* *} P<0.001$.
(B) Quantitation of $\gamma$-H2AX foci in indicated cell lines. Signals co-localizing TRF2 (gray) and those not co-localizing (black) are separately shown. In each condition approximately 70 cells were analyzed for each cell lines.

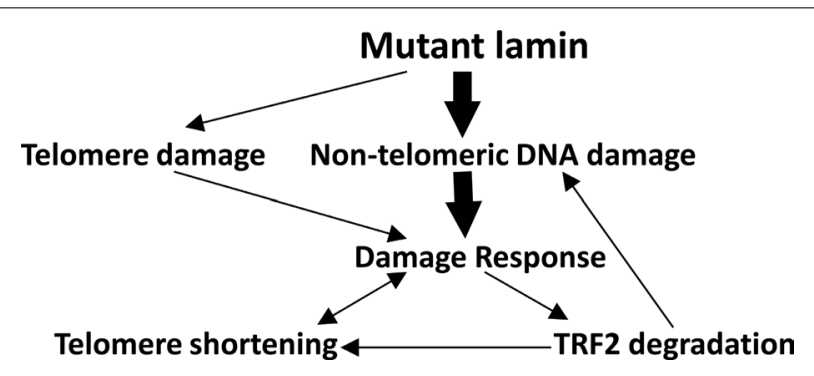

FIGURE 6 | Potential mechanisms of TRF2 degradation and telomere shortening in LMNA mutant AWS cells. Our data support the hypothesis that in young passage cells, though mutant lamin may initiate some DNA damage to the telomeres, the bulk of the DNA damage accumulates within non-telomeric DNA (bold arrows). The latter may be largely responsible for the initiation of a DNA damage response, followed by TRF2 degradation and destabilization of the telomere-shelterin complex. These alterations in turn cause rapid telomere attrition (Oh et al., 2003) and replicative senescence in these cells. Depletion of TRF2 protein along with insufficient DNA damage repair may also increase the overall levels of genomic DNA damage via a positive feedback loop (Mao etal., 2007). In senescent LMNA mutant cells, all the pathways may be activated.

that variations among controls were larger than those between controls and patient cell lines. Therefore we were unable to determine whether accumulation of non-telomeric DNA damage in LMNA mutant cells involves the p53-Siah1 pathways. We observed only a partial stabilization of TRF2 by the addition of proteosomal degradation inhibitor, raising the possibility that other mechanisms of protein degradation may be contributing to the reduction of TRF2 protein in LMNA mutant cells. For example, DNA damage signaling cascades such as p53 and ATM induce autophagy (Hasty et al., 2013). Autophagic activity measured by the LC3-II levels was increased in skeletal muscles and other tissues derived from the Zmpste $24^{-/-}, X P F^{-/-}$, and $C S B / X P A^{-/-}$progeroid mice (Marino et al., 2008). We recently observed increased basal autophagy in LMNA mutant (R133L) fibroblasts used in this study (unpublished data).
We attempted to determine the TRF2 protein half-life using a protein synthesis inhibitor, cycloheximide, in hTERTimmortalized fibroblasts. The previously reported TRF2 protein half-life was longer than 12 h (Nijjar et al., 2005). Our results also indicated that the half-life of TRF2 was greater than $12 \mathrm{~h}$ in both control and LMNA mutant cells (data not shown). We were therefore unable to determine the difference between the protein half-life in LMNA mutant cells as compared to controls. It therefore remains a formal possibility that the reduction of TRF2 in LMNA mutants, at least in part, may be due to a mechanism other than protein instability, such as a decrease in translational efficacy.

Although we have only studied the association of TRF2 with telomeres, there is also a possibility that the binding of other proteins involved in telomere replication or DNA repair factors might be inhibited in LMNA mutant cells, thus contributing to accelerated telomere shortening. The nuclear lamina has been shown to function as a scaffold for chromatin (Broers et al., 2006; Dechat et al., 2008) and various protein complexes (Zastrow et al., 2004) involved in the structural maintenance of the nuclear membrane, gene regulation and signal transduction. In LMNA mutant cells, the disruption of lamina-protein containing complexes and alterations of telomere chromatin structures can affect the accessibility of telomere binding proteins, which could lead to defects in telomere maintenance, including repair and replication of telomeric DNA. Taken together, our data suggest that the decreased level of the shelterin proteins is secondary to the accumulation of DNA damage. The loss of protection of the telomeres can therefore be considered one of the causative factors for the rapid telomere attrition observed in LMNA mutant cells.

There is an increasing amount of evidence for DNA damage and sustained genomic instability as the central cause for laminopathies, including progeroid syndromes such as HGPS (Liu et al., 2005; Scaffidi and Misteli, 2008; Benson et al., 2010; Gonzalez-Suarez and Gonzalo, 2010). Complete loss of lamin A or mutant lamina may directly lead to genomic instability, including 
instability of the telomeres (Liu et al., 2005; Gonzalez-Suarez et al., 2009). Telomere dysfunction then leads to genomic instability, which triggers cellular senescence or apoptosis (Blackburn, 2001; de Lange, 2005). Our study also supports this model, suggesting that unrepaired DNA damage and therefore genomic instability is the common mechanistic cause of the pathophysiology of laminopathies.

\section{REFERENCES}

Andres, V., and Gonzalez, J. M. (2009). Role of A-type lamins in signaling, transcription, and chromatin organization. J. Cell Biol. 187, 945-957. doi: 10.1083/jcb.200904124

Benson, E. K., Lee, S. W., and Aaronson, S. A. (2010). Role of progerin-induced telomere dysfunction in HGPS premature cellular senescence. J. Cell Sci. 123, 26052612. doi: 10.1242/jcs.067306

Blackburn, E. H. (2001). Switching and signaling at the telomere. Cell 106, 661-673. doi: 10.1016/S00928674(01)00492-5

Broers, J. L., Ramaekers, F. C., Bonne, G., Yaou, R. B., and Hutchison, C. J. (2006). Nuclear lamins: laminopathies and their role in premature ageing. Physiol. Rev. 86, 967-1008. doi: 10.1152/physrev.00047.2005

Chen, L., Lee, L., Kudlow, B. A., Dos Santos, H. G., Sletvold, O., Shafeghati, Y., et al. (2003). LMNA mutations in atypical Werner's syndrome. Lancet 362, 440-445. doi: 10.1016/S0140-6736(03)14069-X

Chen, L. Y., Redon, S., and Lingner, J. (2012). The human CST complex is a terminator of telomerase activity. Nature 488, 540-544. doi: 10.1038 /nature 11269

Crabbe, L., Verdun, R. E., Haggblom, C. I., and Karlseder, J. (2004). Defective telomere lagging strand synthesis in cells lacking WRN helicase activity. Science 306, 1951-1953. doi: 10.1126/science.1103619

Dahl, K. N., Scaffidi, P., Islam, M. F., Yodh, A. G., Wilson, K. L., and Misteli, T. (2006). Distinct structural and mechanical properties of the nuclear lamina in Hutchinson-Gilford progeria syndrome. Proc. Natl. Acad. Sci. U.S.A. 103, 10271-10276. doi: 10.1073/ pnas. 0601058103

Dechat, T., Gajewski, A., Korbei, B., Gerlich, D., Daigle, N., Haraguchi, T., et al. (2004). LAP2alpha and BAF transiently localize to telomeres and specific regions on chromatin during nuclear assembly. J. Cell Sci. 117, 6117-6128. doi: 10.1242/jcs.01529

Dechat, T., Pfleghaar, K., Sengupta, K., Shimi, T., Shumaker, D. K., Solimando, L., et al. (2008).
Nuclear lamins: major factors in the structural organization and function of the nucleus and chromatin. Genes Dev. 22, 832-853. doi: $10.1101 / \mathrm{gad} .1652708$

de Lange, T. (2005). Shelterin: the protein complex that shapes and safeguards human telomeres. Genes Dev. 19, 2100-2110. doi: 10.1101/gad.1346005

Denchi, E. L., and de Lange, T. (2007). Protection of telomeres through independent control of ATM and ATR by TRF2 and POT1. Nature 448, 1068-1071. doi: 10.1038/nature06065

De Sandre-Giovannoli, A., Bernard, R., Cau, P., Navarro, C., Amiel, J., Boccaccio, I., et al. (2003). Lamin a truncation in Hutchinson-Gilford progeria. Science 300, 2055. doi: 10.1126/science. 1084125

Eriksson, M., Brown, W. T., Gordon, L. B., Glynn, M. W., Singer, J., Scott, L., et al. (2003). Recurrent de novo point mutations in lamin a cause Hutchinson-Gilford progeria syndrome. Nature 423, 293-298. doi: 10.1038 /nature01629

Friedrich, K., Lee, L., Leistritz, D. F., Nurnberg, G., Saha, B., Hisama, F. M., etal. (2010). WRN mutations in Werner syndrome patients: genomic rearrangements, unusual intronic mutations and ethnicspecific alterations. Hum. Genet. 128, 103-111. doi: 10.1007/s00439-0100832-5

Fujita, K., Horikawa, I., Mondal, A. M., Jenkins, L. M., Appella, E., Vojtesek, B., etal. (2010). Positive feedback between p53 and TRF2 during telomere-damage signalling and cellular senescence. Nat. Cell Biol. 12, 1205-1212. doi: 10.1038/ ncb 2123

Gonzalez-Suarez, I., and Gonzalo, S. (2010). Nurturing the genome: Atype lamins preserve genomic stability. Nucleus 1, 129-135. doi: 10.4161/nucl.1.2.10797

Gonzalez-Suarez, I., Redwood, A. B., Perkins, S. M., Vermolen, B., Lichtensztejin, D., Grotsky, D. A., et al. (2009). Novel roles for Atype lamins in telomere biology and the DNA damage response pathway. $E M B O ~ J . ~ 28,2414-2427$. doi: 10.1038/emboj.2009.196

\section{ACKNOWLEDGMENTS}

We thank Ms. Michelle K. Drews and Mr. Alex Cypro for the editorial assistance and Dr. Lisa A. Lai for the technical assistance for immuno-FISH data analyses. This work was supported by $\mathrm{NIH}$ grants, R24 CA78088, R24 AG42328, and R21 AG42879, the Ellison Medical Foundation Senior Scholar Award and the American Heart Association fellowship.

Hansen, R. S., Thomas, S., Sandstrom, R., Canfield, T. K., Thurman, R. E., Weaver, M., et al. (2010). Sequencing newly replicated DNA reveals widespread plasticity in human replication timing. Proc. Natl. Acad. Sci. U.S.A. 107, 139-144. doi: 10.1073/pnas.0912402107

Hasty, P., Sharp, Z. D., Curiel, T. J., and Campisi, J. (2013). mTORC1 and p53: clash of the gods? Cell Cycle 12, 20-25. doi: 10.4161/cc.22912

Hisama, F. M., Lessel, D., Leistritz, D., Friedrich, K., McBride, K. L., Pastore, M. T., et al. (2011). Coronary artery disease in a Werner syndromelike form of progeria characterized by low levels of progerin, a splice variant of lamin A. Am. J. Med. Genet. A 155A, 3002-3006. doi: 10.1002/ajmg.a.34336

Huang, S., Risques, R. A., Martin, G. M., Rabinovitch, P. S. and Oshima, J. (2008). Accelerated telomere shortening and replicative senescence in human fibroblasts overexpressing mutant and wild-type lamin A. Exp. Cell Res. 314, 82-91. doi: 10.1016/j.yexcr.2007.08.004

Kudlow, B. A., Stanfel, M. N., Burtner, C. R., Johnston, E. D., and Kennedy, B. K. (2008). Suppression of proliferative defects associated with processing-defective lamin A mutants by hTERT or inactivation of p53. Mol. Biol. Cell 19, 5238-5248. doi: 10.1091/mbc.E08-05-0492

Liu, B., Wang, J., Chan, K. M., Tjia, W. M., Deng, W., Guan, X., et al. (2005). Genomic instability in laminopathybased premature aging. Nat. Med. 11 , 780-785. doi: 10.1038/nm1266

Liu, Y., Rusinol, A., Sinensky, M., Wang, Y., and Zou, Y. (2006). DNA damage responses in progeroid syndromes arise from defective maturation of prelamin A. J. Cell Sci. 119 4644-4649. doi: 10.1242/jcs.03263

Luderus, M. E., van Steensel, B., Chong, L., Sibon, O. C., Cremers, F. F., and de Lange, T. (1996). Structure, subnuclear distribution, and nuclear matrix association of the mammalian telomeric complex. J. Cell Biol. 135 , 867-881. doi: 10.1083/jcb.135.4.867

Mao, Z., Seluanov, A., Jiang, Y., and Gorbunova, V. (2007). TRF2 is required for repair of nontelomeric DNA double-strand breaks by homologous recombination. Proc. Natl. Acad. Sci. U.S.A. 104, 13068-13073. doi: 10.1073/pnas.0702410104

Marino, G., Ugalde, A. P., SalvadorMontoliu, N., Varela, I., Quiros, P. M., Cadinanos, J., et al. (2008). Premature aging in mice activates a systemic metabolic response involving autophagy induction. Hum. Mol. Genet. 17, 2196-2211. doi: $10.1093 / \mathrm{hmg} / \mathrm{ddn} 120$

Martin, G. M. (1978). Genetic syndromes in man with potential relevance to the pathobiology of aging. Birth Defects Orig. Artic. Ser. 14, 5-39. Musich, P. R., and Zou, Y. (2011). DNA-damage accumulation and replicative arrest in HutchinsonGilford progeria syndrome. Biochem. Soc. Trans. 39, 1764-1769. doi: 10.1042/BST20110687

Nijjar, T., Bassett, E., Garbe, J., Takenaka, Y., Stampfer, M. R., Gilley, D., et al. (2005). Accumulation and altered localization of telomere-associated protein TRF2 in immortally transformed and tumor-derived human breast cells. Oncogene 24, 3369-3376. doi: 10.1038/sj.onc. 1208482

Oh, H., Wang, S. C., Prahash, A., Sano, M., Moravec, C. S., Taffet, G. E., et al. (2003). Telomere attrition and Chk2 activation in human heart failure. Proc. Natl. Acad. Sci. U.S.A. 100, 5378-5383. doi: 10.1073/pnas.0836098100

Okamoto, K., Bartocci, C., Ouzounov, I., Diedrich, J. K., Yates, J. R. III, and Denchi, E. L. (2013). A two-step mechanism for TRF2mediated chromosome-end protection. Nature 494, 502-505. doi: 10.1038/nature11873

Oshima, J., Campisi, J., Tannock, T. C., and Martin, G. M. (1995). Regulation of c-fos expression in senescing Werner syndrome fibroblasts differs from that observed in senescing fibroblasts from normal donors. J. Cell. Physiol. 162, 277-283. doi: 10.1002/jcp. 1041620213

O’Sullivan, J. N., Finley, J. C., Risques, R. A., Shen, W. T., Gollahon, K. A., Moskovitz, A. H., et al. (2004). Telomere length assessment in tissue sections by quantitative FISH: image analysis algorithms. Cytometry $A$ 58, 120-131. doi: 10.1002/cyto.a.20006 
Palm, W., and de Lange, T. (2008). How shelterin protects mammalian telomeres. Annu. Rev. Genet. 42, 301334. doi: 10.1146/annurev.genet.41. 110306.130350

Rubio, M. A., Kim, S. H., and Campisi, J. (2002). Reversible manipulation of telomerase expression and telomere length. Implications for the ionizing radiation response and replicative senescence of human cells. J. Biol. Chem. 277, 28609-28617. doi: 10.1074/jbc.M203747200

Saha, B., Lessel, D., Hisama, F. M., Leistritz, D. F., Friedrich, K., Martin, G. M., etal. (2010). A novel LMNA mutation causes altered nuclear morphology and symptoms of familial partial lipodystrophy (Dunnigan variety) with progeroid features. Mol. Syndromol. 1, 127-132. doi: $10.1159 / 000320166$

Scaffidi, P., and Misteli, T. (2006). Lamin A-dependent nuclear defects in human aging. Science 312, 1059 1063. doi: 10.1126/science. 1127168
Scaffidi, P., and Misteli, T. (2008). Lamin A-dependent misregulation of adult stem cells associated with accelerated ageing. Nat. Cell Biol. 10, 452-459. doi: $10.1038 /$ ncb 1708

Sfeir, A., and de Lange, T. (2012). Removal of shelterin reveals the telomere end-protection problem. Science 336, 593-597. doi: 10.1126/science. 1218498

Shumaker, D. K., Dechat, T., Kohlmaier, A., Adam, S. A., Bozovsky, M. R., Erdos, M. R., et al. (2006). Mutant nuclear lamin A leads to progressive alterations of epigenetic control in premature aging. Proc. Natl. Acad. Sci. U.S.A. 103, 8703-8708. doi: 10.1073/pnas.0602569103

Suram, A., Kaplunov, J., Patel, P. L. Ruan, H., Cerutti, A., Boccardi, V., et al. (2012). Oncogene-induced telomere dysfunction enforces cellular senescence in human cancer precursor lesions. EMBO J. 31, 2839-2851. doi: 10.1038/emboj. 2012.132
Yu, C. E., Oshima, J., Fu, Y. H., Wijsman, E. M., Hisama, F., Alisch, R., etal. (1996). Positional cloning of the Werner's syndrome gene. Science 272, 258-262. doi: 10.1126/science. 272.5259 .258

Zastrow, M. S., Vlcek, S., and Wilson, K. L. (2004). Proteins that bind A-type lamins: integrating isolated clues. J. Cell Sci. 117, 979-987. doi: 10.1242/jcs.01102

Zhou, W. J., Deng, R., Zhang, X. Y., Feng, G. K., Gu, L. Q., and Zhu, X. F. (2009). G-quadruplex ligand SYUIQ5 induces autophagy by telomere damage and TRF2 delocalization in cancer cells. Mol. Cancer Ther 8, 3203-3213. doi: 10.1158/15357163.MCT-09-0244

Conflict of Interest Statement: The authors declare that the research was conducted in the absence of any commercial or financial relationships that could be construed as a potential conflict of interest.

Received: 12 February 2013; accepted: 16 June 2013; published online: 05 July 2013.

Citation: Saha B, Zitnik G, Johnson S, Nguyen Q, Risques RA, Martin GM and Oshima J (2013) DNA damage accumulation and TRF2 degradation in atypical Werner syndrome fibroblasts with LMNA mutations. Front. Genet. 4:129. doi: 10.3389/fgene.2013.00129

This article was submitted to Frontiers in Genetics of Aging, a specialty of Frontiers in Genetics.

Copyright (C) 2013 Saha, Zitnik, Johnson, Nguyen, Risques, Martin and Oshima. This is an open-access article distributed under the terms of the Creative Commons Attribution License, which permits use, distribution and reproduction in other forums, provided the original authors and source are credited and subject to any copyright notices concerning any third-party graphics etc. 